This item was submitted to Loughborough's Research Repository by the author.

Items in Figshare are protected by copyright, with all rights reserved, unless otherwise indicated.

\title{
The use of rapid prototyping in the design of a customised ankle brace structure for ACL injury risk reduction.
}

PLEASE CITE THE PUBLISHED VERSION

http://dx.doi.org/10.1080/17452759.2013.863533

\section{PUBLISHER}

(c) Taylor and Francis

\section{VERSION}

AM (Accepted Manuscript)

\section{PUBLISHER STATEMENT}

This work is made available according to the conditions of the Creative Commons Attribution-NonCommercialNoDerivatives 4.0 International (CC BY-NC-ND 4.0) licence. Full details of this licence are available at: https://creativecommons.org/licenses/by-nc-nd/4.0/

\section{LICENCE}

CC BY-NC-ND 4.0

\section{REPOSITORY RECORD}

Teng, Phillip S.P., K.F. Leong, Pui W. Kong, Ben J. Halkon, and P.Y. Huang. 2019. "The Use of Rapid Prototyping in the Design of a Customised Ankle Brace Structure for ACL Injury Risk Reduction.”. figshare. https://hdl.handle.net/2134/15891. 


\title{
The Use of Rapid Prototyping in the Design of a Customized Ankle Brace Structure for ACL Injury Risk Reduction
}

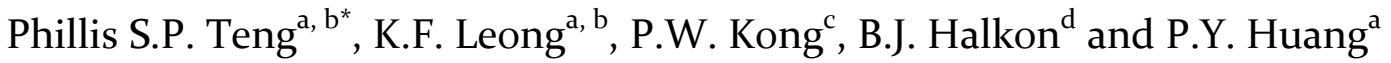 \\ ${ }^{a}$ School of Mechanical and Aerospace Engineering, Nanyang Technological University, Singapore \\ ${ }^{b}$ Institute for Sports Research, Nanyang Technological University, Singapore \\ ${ }^{c}$ Physical Education and Sports Science Academic Group, National Institute of Education, Nanyang Technological \\ University, Singapore \\ ${ }^{d}$ Wolfson School of Mechanical and Manufacturing Engineering, Loughborough University, United Kingdom \\ *Corresponding author. Email: phillis.teng@ntu.edu.sg
}

\begin{abstract}
Rapid prototyping, or additive manufacturing, is becoming more useful in creating functional prototypes, especially when customization is required. This paper explores the use of three dimensional $(3 \mathrm{D})$ printing in designing a customized ankle brace structure for Anterior Cruciate Ligament (ACL) injury risk reduction. A new process is proposed to obtain ankle flexion angles and the corresponding foot surface strain associated with high ACL injury risks through motion analysis. This data is used in the design of the customized ankle brace structure and printed using rapid prototyping. One customized ankle brace structure was printed and tested to demonstrate this proposed framework. The ankle flexion range of motion (ROM) was significantly reduced in the high-risk ankle positions with the ankle brace structure. Rapid prototyping could thus be used to design customized ankle brace structures and this is useful in reducing fabrication time and complexity of customization.
\end{abstract}

Keywords: anterior cruciate ligament, rapid prototyping, additive manufacturing, customized ankle brace structure

\section{Introduction}

Rapid prototyping (RP), or additive manufacturing, can easily be used for customization of functional prototypes, without the need for costly moulds producing small volumes to meet tailored requirements. Besides, time to manufacture is usually shorter and has been proposed for customized medical applications (De Crescenzio et al. 2011, Mavroidis et al. 2011, Cheng and Chu 2013). In this paper, the use of RP is also proposed for a new framework to design a customized ankle brace structure for Anterior Cruciate Ligament (ACL) injury risk reduction.

ACL injury has a significant impact on the performance and health of athletes. It takes almost 8 months after operation to return to competition and on average, 5 years to reach pre-injury performance (Roi et al. 2006). Besides, ACL injuries also result in high financial costs with ACL surgery costs in the United States estimating to be more than \$2 billion each year (Ali and Rouhi 2010). Furthermore, incidence of ACL injuries continues to rise (Donnelly et al. 2012) and hence continual effort is required to help reduce ACL injury risks among athletes.

Most ACL injuries happen in non-contact situations without contact with another object or player (Ali and Rouhi 2010). A low knee flexion angle during landing is associated with a higher non-contact ACL injury risk (Lin et al. 2008). Video analyses had also identified low knee flexion angles just before ACL injuries took place (Lin et al. 2008). In addition, one cadaver study also found that the highest in-situ ACL force was at $15^{\circ}$ knee flexion ( $\mathrm{Li}$ et al. 1999). A knee constraint brace was also designed to provide resistance to prevent athletes from landing at low knee flexion angles (Lin et al. 2008). However, some healthy athletes are still not willing to wear a knee brace for fear of a drop in performance (Rishiraj et al. 2009). This proposed framework also makes use of data from a new study to understand how knee flexion angles can be increased from a foot landing and ankle brace perspective. Healthy athletes have used ankle braces for sprain prevention (Shaw et al. 2008) and its use could be further extended for ACL injury risk reduction. Higher plantar flexion angles were found to result in lower knee flexion angles at initial contact during jump-landing (Cortes et al. 2007). ACL strain was also found to be the highest at initial contact with the ground during jump-landing (Lamontagne et al. 2008). Thus while landing toe-first is associated with greater force absorption (Alentorn-Geli et al. 2009), too high a plantar-flexion landing might potentially reduce knee flexion angles to $15^{\circ}$ which could result in high ACL loading. Therefore, in this proposed framework, the ankle brace structure is used to resist motion of the ankle associated with $15^{\circ}$ of knee flexion angle to prevent the onset of the high ACL loading. 


\section{Methods}

The proposed framework to design a customised ankle brace structure is illustrated in Figure 1. The purpose of the ankle brace structure is for it to be incorporated as a part of an ankle brace to prevent the foot from going into positions associated with high ACL injury risks and

to allow little resistance in foot positions associated with low ACL injury risks of each individual.

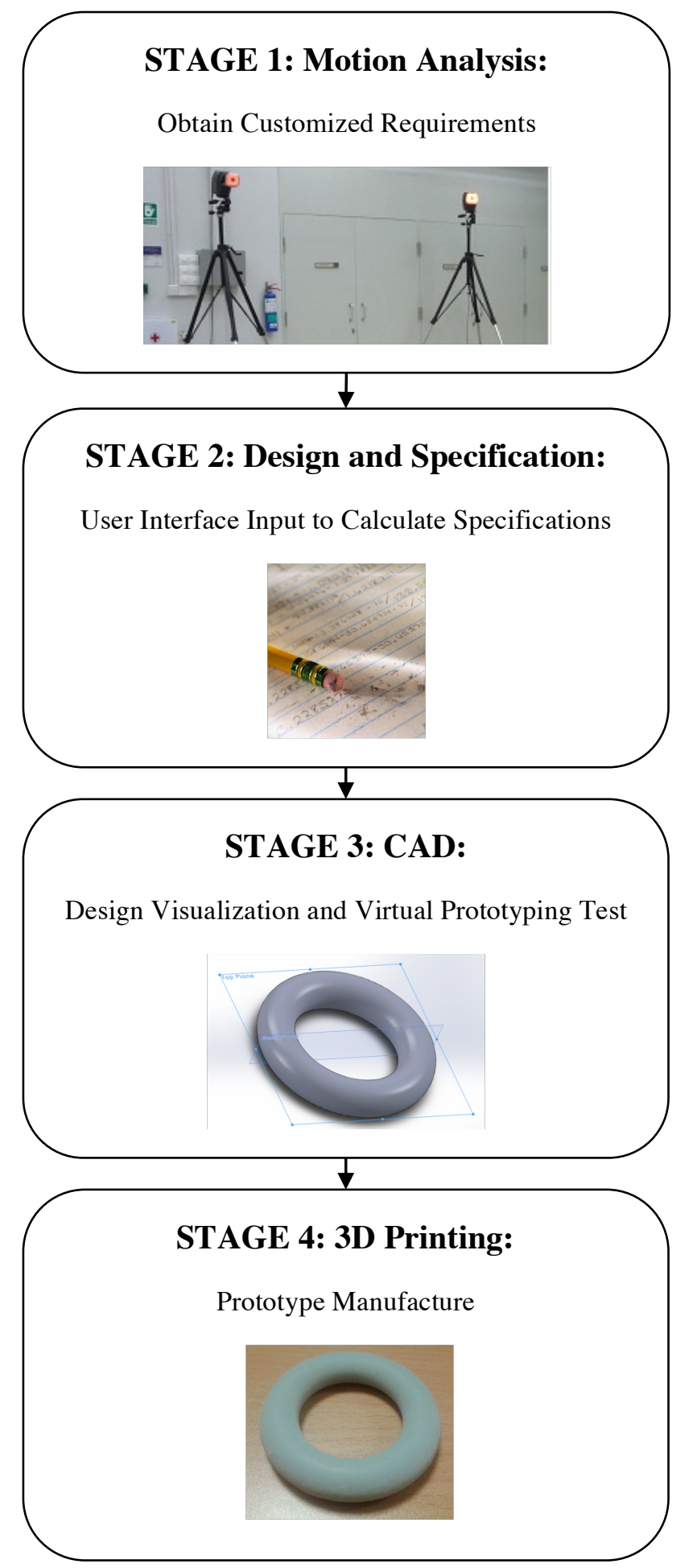

Figure 1. Proposed flowchart for design of customized ankle brace structure

The ankle brace structure is proposed to be manufactured using $3 \mathrm{D}$ printing with the use of flexible materials and a donut geometrical shape is being used for the purpose of this feasibility study. At low risk positions, there will be little resistance in the donut geometrical shape to allow the full range of motion during play (Region 'A' of Figure 2) as the structure will flex freely. When the material reaches the threshold strain, the donut geometrical shape will become fully stretched (Region 'B' of Figure 2). It will go into a different stressstrain curve and there will be a resistive force as a result of the tensile properties of the material. This will resist motion of the ankle to prevent the onset of the high

ACL loading as the ankle reaches the critical plantar/dorsi flexion angle. The ankle brace structure will be found at the location on the foot that will experience the maximum strain during plantar/dorsi flexion. This will be determined for each individual.

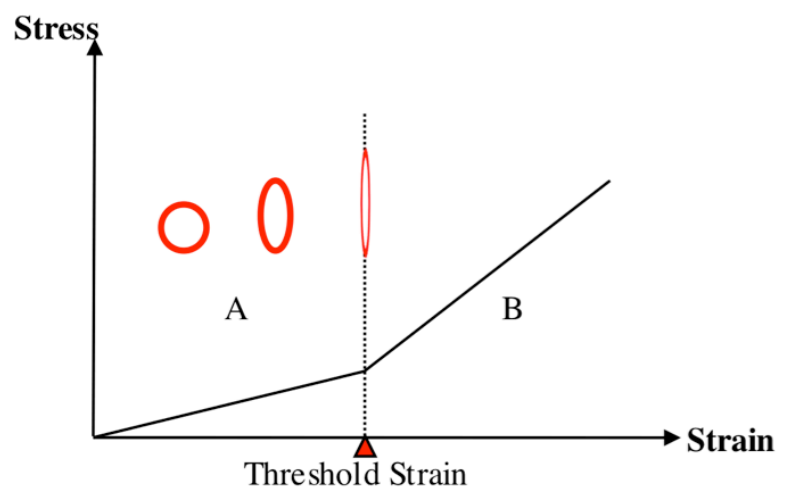

Figure 2. Stress-strain graph and the changes to the ankle brace structure

Motion analysis capture is first carried out to obtain the critical plantar flexion angle associated with $15^{\circ}$ of knee flexion angle (Figure 1, stage 1). Strain study is also carried out using the motion analysis capture to obtain the strain distribution of the foot surface when the ankle flexes to the critical plantar flexion angle (Figure 1, stage 1). This provides the individual measurement for each person that could subsequently be input into a prewritten program with a user interface that easily calculates specifications for each indi- vidual (Figure 1, stage 2). Computer aided design (CAD) is then used to create the model of the ankle brace structure to be printed by 3D printing (Figure 1, stage 3). Virtual prototyping (VP) could subsequently be added to ensure fit before printing the functional model (Figure 1, stage 1). The ankle brace structure can then be printed using ${ }_{3} \mathrm{D}$ printing (Figure 1, stage 4). A proof-of-concept prototype was built in this paper using $3 \mathrm{D}$ printing to demonstrate this proposed framework. Range of motion tests were carried out to study the feasibility of using this proposed framework.

\section{Motion Analysis Capture}

\section{Participants}

Six male subjects (age: mean 23.5 (SD 2.6) years old, height: mean 1.77 (SD 0.05) m, weight: mean 72.7 (SD $7.8) \mathrm{kg}$ ) participated in this study. Participants had no injuries in the last 6 months before the study. The research was approved by the Institutional Review Board of the university. Each participant also signed a written consent form to take part in the study. 


\section{Markers and Motion Analysis System}

Retroreflective markers were placed on the greater trochanter, lateral knee epicondyle, lateral malleolus, heel and toe (between second and third metatarsals) of the right leg (Figure 3a). The motion analysis system consisted of 7 digital cameras (Motion Analysis Corp, Santa Rosa, CA, USA) and was sampled at $240 \mathrm{~Hz}$. Motion capture data was collected using Cortex (version 1.1.4.368, Motion Analysis Corp, Santa Rosa, CA, USA) and two-dimensional kinematic calculations were used to calculate the knee flexion and plantar-dorsi flexion angles, as defined in Figure 3b. Marker data was filtered through a low-pass Butterworth digital filter at a cut-off frequency of $9 \mathrm{~Hz}$. Knee flexion and dorsi flexion angles were denoted as positive.

(a) Marker Positions

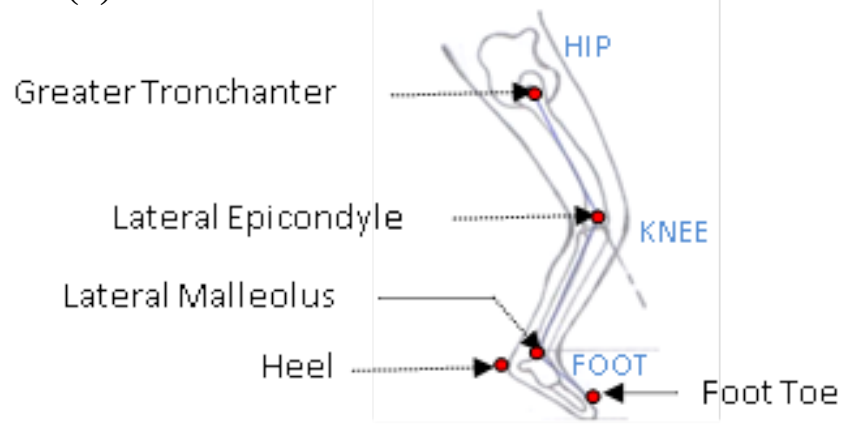

Test Protocol

Subjects were requested to perform drop vertical jumps (DVJ) from a platform ( $31 \mathrm{~cm}$ in height) with their feet $35 \mathrm{~cm}$ apart. Subjects were asked to step off the platform and to land with both feet on the ground. Immediately after, subjects were then asked to jump to as

high as possible. The first jump from the platform was used for the analysis.

\section{Critical Plantar Flexion Angle}

Knee flexion angles were plotted in the y axis and corresponding plantar-dorsi flexion angles were plotted in the $\mathrm{x}$ axis. $\mathrm{A} 2^{\text {nd }}$-order polynomial was plotted and a quadratic equation with the $r^{2}$ value of 0.8 was obtained. Results are shown in Figure 4. At $15^{\circ}$ of knee flexion angle, a critical plantar flexion angle was calculated to be around $-33^{\circ}$.

Figure 3. (a) Positions of markers on the bony protrusions at the outer part of the right leg (on the greater trochanter at the hip, lateral epicondyle at the thigh bone, lateral malleolus at the ankle, at the heel and foot toe) and (b) definition of knee flexion and ankle flexion angles

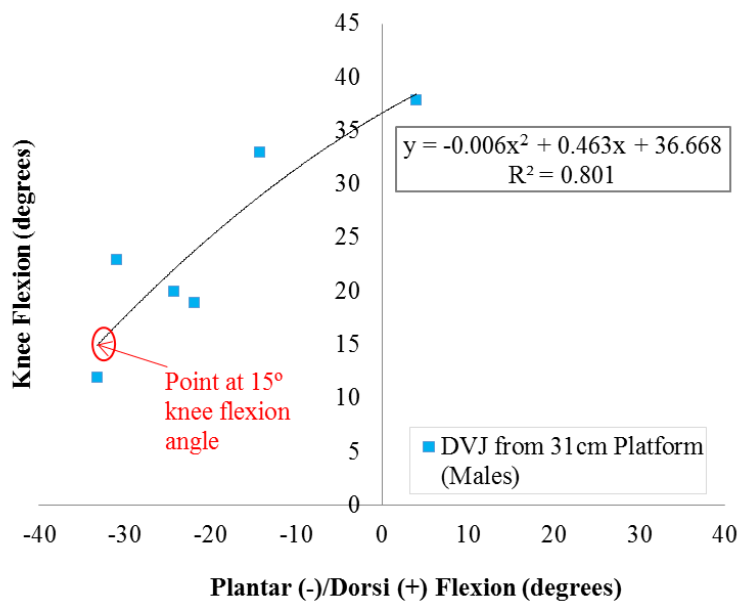

Figure 4. Plot of knee flexion versus ankle flexion angles at initial contact

\section{Strain Study during Plantar Flexion}

Markers and Motion Analysis System

An array of markers was placed on the foot to study the local strain distribution. Motion analysis capture allows kinematic data to be obtained throughout the dynamic motion. In this proof-of-concept phase, 7 markers were placed on the top part of the foot of one subject. Figure 5 shows the location of the markers on the foot. The lateral 2 markers ('A' and 'B') were used to measure the strain at the lateral region of the foot. The centre 3 markers ('C' to 'E') were used to measure the strains at the centre region of the foot. The medial 2 markers (' $F$ ' and ' $G$ ') were used to measure the strain at the medial region of the foot. The motion analysis system consisted of 7 digital cameras (Motion Analysis Corp, Santa Rosa, CA, USA) and was sampled at $240 \mathrm{~Hz}$. Motion capture data was collected using Cortex (version 1.1.4.368, Motion Analysis Corp, Santa Rosa, CA, USA). Marker data was once again filtered through a low-pass Butterworth digital filter at a cut-off frequency of $9 \mathrm{~Hz}$.

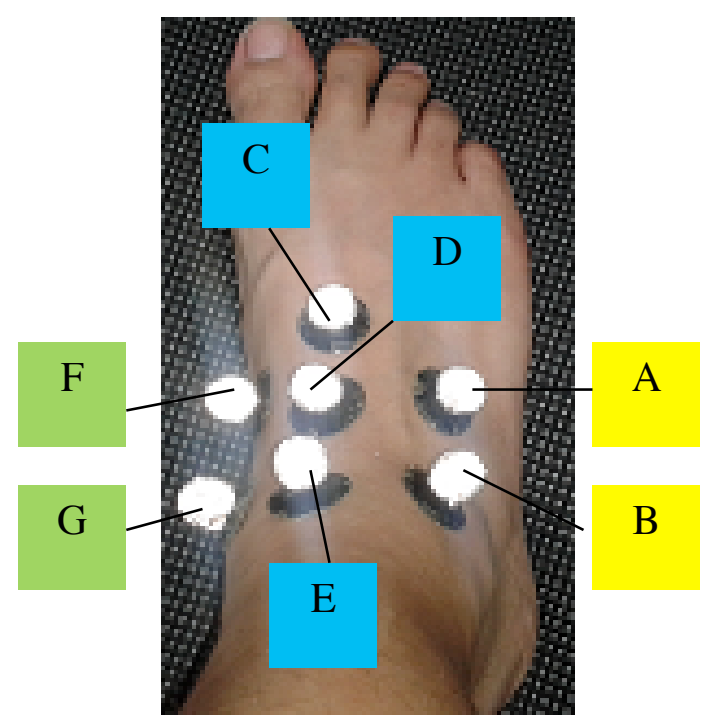

Figure 5. Position of markers on the foot 


\section{Strain Calculation}

The marker positions were first recorded at rest position. Each marker position was at coordinates (x, y, z). With ankle flexion, each marker attained a new coordinate position ( $\left.\mathrm{x}^{\prime}, \mathrm{y}^{\prime}, \mathrm{z}^{\prime}\right)$. Thus, distance between 2 marker positions at rest position was calculated as:
Distance $L=\sqrt{\left(\mathrm{x}_{1}-\mathrm{x}_{2}\right)^{2}+\left(\mathrm{y}_{1}-\mathrm{y}_{2}\right)^{2}+\left(\mathrm{z}_{1}-\mathrm{z}_{2}\right)^{2}}$

Distance between the $\mathbf{2}$ markers after ankle flexion is:

Local strain between 2 markers due to the motion is:

$$
\frac{\Delta L}{L}=\frac{\sqrt{\left(x_{1}^{\prime}-x_{2}^{\prime}\right)^{2}+\left(y_{1}^{\prime}-y_{2}^{\prime}\right)^{2}+\left(z_{1}^{\prime}-z_{2}^{\prime}\right)^{2}}-\sqrt{\left(x_{1}-x_{2}\right)^{2}+\left(y_{1}-y_{2}\right)^{2}+\left(z_{1}-z_{2}\right)^{2}}}{\sqrt{\left(x_{1}-x_{2}\right)^{2}+\left(y_{1}-y_{2}\right)^{2}+\left(z_{1}-z_{2}\right)^{2}}}
$$

The local strain between markers was obtained from the above equations. For this prototype, the assumption was that the highest strain occurred along the longitudinal direction of the foot (A-B, C-D-E and F-G direction of the foot in Figure 5 ).

\section{Test Protocol}

Subject was requested to plantar-flex and then dorsiflex the ankle to as much as possible.

Design of the Ankle Brace Structure for $3 \mathrm{D}$ Printing

Data was plotted between surface strain (y-axis) and plantar-dorsi flexion angles ( $\mathrm{x}$-axis). Dorsi flexion angle was denoted as positive. A $2^{\text {nd }}$-order polynomial was plotted and strain value at $-33^{\circ}$ critical plantar flexion angle was calculated. The highest strain (58\%) was used in the design of the ankle brace structure and the graph was plotted in Figure 6 . The strain value was used to design the diameter of the donut while the crosssectional area was estimated from the forces experienced by the foot measured during landing and tensile strength of the material. This is illustrated in Figure 7.

\section{Rapid Prototyping}

Prototype was produced with the $3 \mathrm{D}$ printer by Objet ${ }^{\circledast}$ Eden35oV (Objet Geometries Ltd., Rehovot, Isreal) using the material TangoGray ${ }^{\mathrm{TM}}$ FLX950 and supporting material Support SUP705. The ankle brace structure in the form of a donut shape was made and is shown in Figure 8 with a leg model.

\section{Range of Motion Test}

\section{Markers and Motion Analysis System}

Retroreflective markers were placed on the lateral and medial malleoli, heel and toe (between second and third metatarsals) of the right leg. The motion analysis system consisted of 7 digital cameras (Motion Analysis Corp, Santa Rosa, CA, USA) and was sampled at $24 \mathrm{oHz}$. Motion capture data was collected using Cortex (version 1.1.4.368, Motion Analysis Corp, Santa Rosa, CA, USA) and two-dimensional kinematic calculations were used to calculate plantar-dorsi flexion angles. Marker data was filtered through a low-pass Butterworth digital filter at a cut-off frequency of $9 \mathrm{~Hz}$. Dorsi flexion angles were denoted as positive.

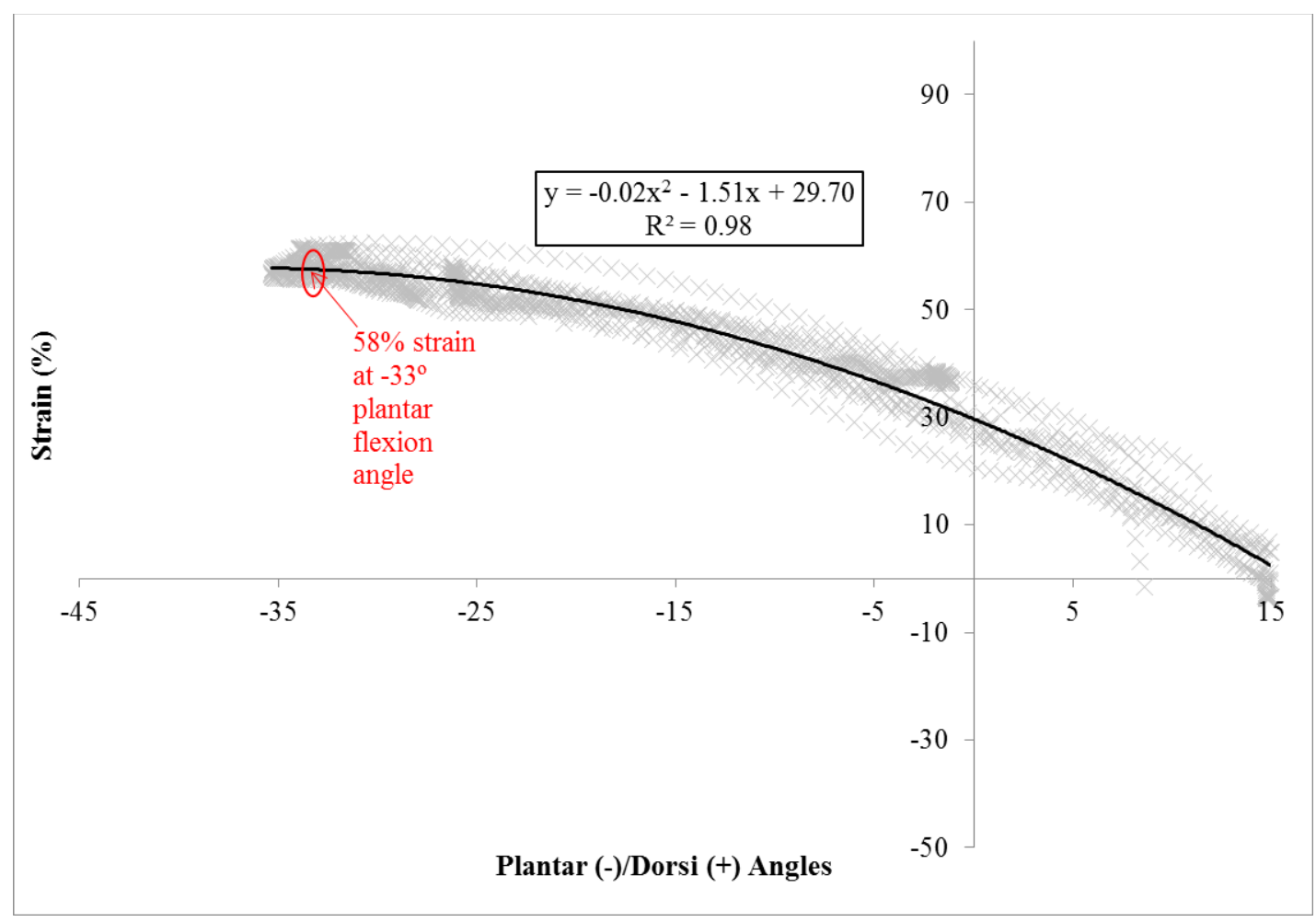

Figure 6. Plot of foot surface strain against plantar flexion angle 


\section{Determination of Diameter}
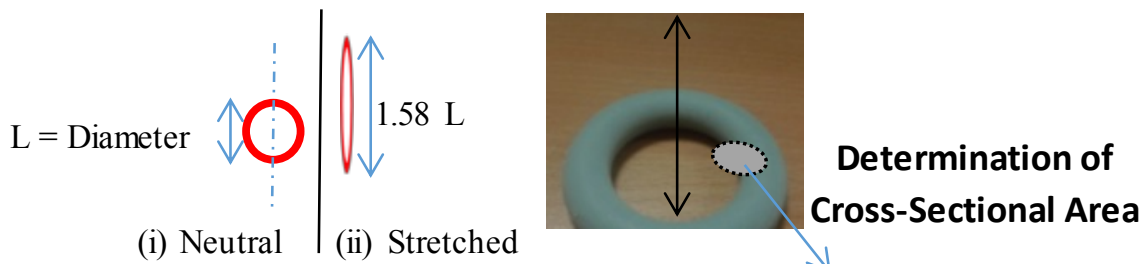

Cross-sectional area $(\mathrm{A})$

estimated from tensile strength of material $\left(\sigma_{\mathrm{TS}}\right)$ and forces $(\mathrm{F})$ experienced by the foot during landing:

$\sigma_{\mathrm{TS}} /$ Safety Factor $=\mathrm{F}^{\prime} / \mathrm{A}$

where $F^{\prime}=F / 2$ shared by

the two sides of the

structure when stretched

Figure 7. Design of the ankle brace structure

\section{Test Protocol}

The subject was requested to plantar-flex and then to dorsi-flex the ankle to as much as possible. This was repeated with the ankle brace structure worn. Six independent trials were conducted for each condition.

Structure was secured with string to the foot in this feasibility study and is illustrated with a leg model in

Figure 8.

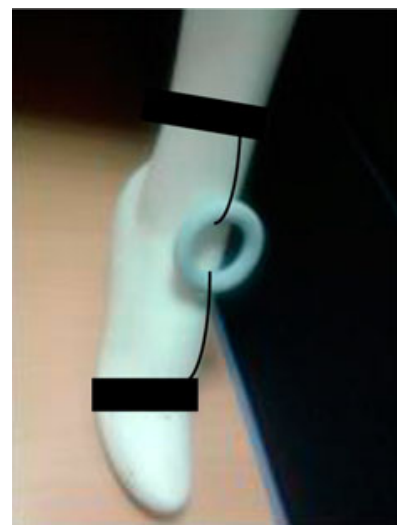

Figure 8. Prototype ankle brace structure on a leg model

\section{Software}

SolidWorks ${ }^{\circledast}$ (Dassault Systèmes SolidWorks Corporation, Waltham, MA, USA) was used to design the CAD model of the ankle brace structure.

Statistical analyses were conducted using Minitab (version 16, Minitab Inc., State College, Pa, USA). Wilcoxon signed rank test was used and the level of significance was 0.05 .

\section{Results and Discussion \\ Range of Motion (ROM) Test}

Results show that without any brace, the ankle can be plantar flexed to 55.5 (SD o.2) - while it can only be flexed to 44.9 (SD 3.7)o with the ankle brace structure. Wilcoxon signed rank test (with $\alpha=0.05$ ) showed sig- nificant differences in the 2 ranges of motion, supporting that the ankle brace structure was causing a significant reduction in the ROM. Figure 9 shows the box plots of the results.

The structure was able to reduce the range of motion and to resist motion in the high plantar flexion region associated with knee flexion angles less than $15^{\circ}$. At knee flexion angles greater than $1^{\circ}$, the ankle was free to move. This prototype result shows that rapid prototyping could potentially be used to design customized ankle brace structures to help resist motion associated with high ACL injury risks. Strain distribution on the foot surface may differ among people due to their body fats. Customization could easily be carried out and tailored specifications can easily be calculated using a prewritten program. The structure was designed to start resisting motion from $-33^{\circ}$ and a dynamic jump-land test could be conducted in future to further analyze if angular deceleration occurred close to $-33^{\circ}$.

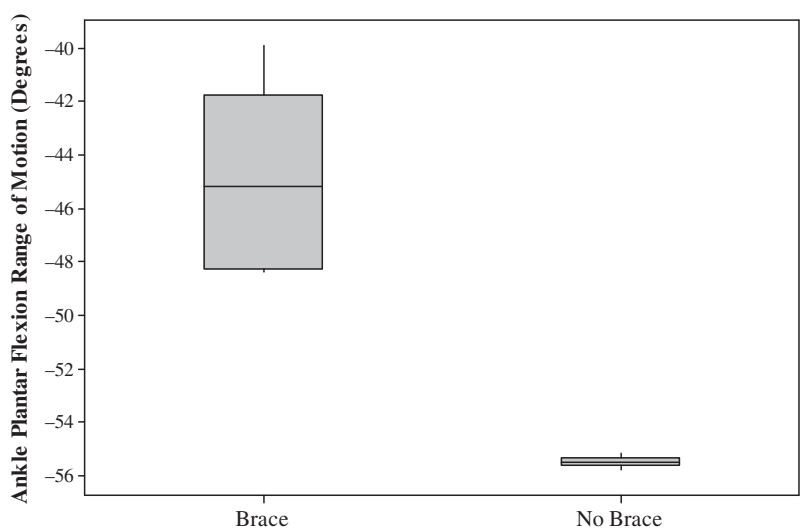

Figure 9. Box plot of ankle plantar range of motion under 'brace' and 'no brace' conditions

\section{Time to Manufacture}

Stages 1 and 2 of Figure 1 are the same, with or without the use of RP or VP. However, stages 3 and 4 have been made easier with the use of VP and RP. Although not 
carried out for this feasibility test, VP could be introduced in Stage 3 to verify the functional fit or range of motion of each individual before it is actually printed. The $3 \mathrm{D}$ printing in stage 4 of Figure 1 is easily carried out in $1.5 \mathrm{hr}$. Alternatively, moulding could be carried out but this will increase manufacture time and costs. Besides, RP could allow greater customization flexibility in terms of size and shape fit. It will not be financially practical to make one mould for every personalized size.

\section{Reduction in Design Complexity}

In this feasibility study, the simple donut structure was adopted. However, more design structure shapes could be easily and quickly explored using VP and RP. Diamond geometrical shapes would be explored in future as this shape will allow for greater flexibility in the low resistance region. Future designs would focus on smaller and flatter designs that could easily be worn. It would otherwise be costly and time-consuming to manufacture few parts using temporary moulds at each prototype design stage. This would greatly reduce product development iteration time.

\section{Conclusions}

A framework to customize ankle brace structures for ACL injury risk reduction with the use of RP has been proposed. RP can quickly and easily manufacture functional prototypes for customized specifications for individuals. In this feasibility study, a prototype was also produced and results show that the brace structure was able to reduce ROM in the high-risk ankle positions as designed. Although more design refinement is required for the structural shape, this result shows that RP could potentially be used in customized ankle brace structures for use in ACL injury risk reduction.

\section{References}

Alentorn-Geli, E., Myer, G. D., Silvers, H. J., Samitier, G., Romero, D., Lázaro-Haro, C. \& Cugat, R., 20o9. Prevention of non-contact anterior cruciate ligament injuries in soccer players. Part 1: mechanisms of injury and underlying risk factors. Knee Surgery, Sports Traumatology, Arthroscopy, 17 (7), 705-729.

Ali, N. \& Rouhi, G., 2010. Barriers to predicting the mechanisms and risk factors of non-contact anterior cruciate ligament injury. The Open Biomedical Engineering Journal, 4 178-189.
Cheng, Y.-L. \& Chu, J.-C., 2013. Application of rapid tooling to manufacture customized nasal mask cushion for continuous positive airway pressure (CPAP) devices. Rapid Prototyping Journal, 19 (1), 4-10.

Cortes, N., Onate, J., Abrantes, J., Gagen, L., Dowling, E. \& Van Lunen, B., 2007. Effects of gender and foot-landing techniques on lower extremity kinematics during drop-jump landings. Journal of Applied Biomechanics, 23 (4), 289-299.

De Crescenzio, F., Fantini, M., Ciocca, L., Persiani, F. \& Scotti, R., 2011. Design and manufacturing of ear prosthesis by means of rapid prototyping technology. Proceedings of the Institution of Mechanical Engineers, Part H: Journal of Engineering in Medicine, $225\left(\mathrm{H}_{3}\right)$, 296-302.

Donnelly, C. J., Elliott, B. C., Ackland, T. R., Doyle, T. L. A., Beiser, T. F., Finch, C. F., Cochrane, J. L., Dempsey, A. R. \& Lloyd, D. G., 2012. An anterior cruciate ligament injury prevention framework: incorporating the recent evidence. Research in Sports Medicine, 20 (3/4), 239-262.

Lamontagne, M., Benoit, D. L., Ramsey, D. K., Caraffa, A. \& Cerulli, G., 2008. In vivo biomechanical study for injury prevention. In: Y. Hong and R. Bartlett, eds. Handbook of biomechanics and human movement science. London; New York: Routledge, 428-445.

Li, G., Rudy, T. W., Sakane, M., Kanamori, A., Ma, C. B. \& Woo, S. L.-Y., 1999. The importance of quadriceps and hamstring muscle loading on knee kinematics and in-situ forces in the ACL. Journal of Biomechics, 32 (4), 395-40o.

Lin, C.-F., Liu, H., Garrett, W. E. \& Yu, B., 2008. Effects of a knee extension constraint brace on selected lower extremity motion patterns during a stop-jump task. Journal of Applied Biomechanics, 24 (2), 158-165.

Mavroidis, C., Ranky, R. G., Sivak, M. L., Patritti, B. L., DiPisa, J., Caddle, A., Gilhooly, K., Govoni, L., Sivak, S., Lancia, M., Drillio, R. \& Bonato, P., 2011. Patient specific ankle-foot orthoses using rapid prototyping. Journal of Neuroengineering and Rehabilitation, 8 (1), 11p.

Rishiraj, N., Taunton, J. E., Lloyd-Smith, R., Woollard, R., Regan, W. \& Clement, D. B., 2009. The potential role of prophylactic/functional knee bracing in preventing knee ligament injury. Sports Medicine, 39 (11), 937-96o.

Roi, G. S., Nanni, G. \& Tencone, F., 20o6. Time to return to professional soccer matches after ACL reconstruction. Sport Sciences for Health, 1 (4), 142-145.

Shaw, M. Y., Gribble, P. A. \& Frye, J. L., 2008. Ankle bracing, fatigue, and time to stabilization in collegiate volleyball athletes. Journal of Athletic Training, 43 (2), 164-171 